\title{
An Ultra-Flat Frequency Comb Generated Using Nonlinear Broadening and Adaptive Pulse Shaping
}

\author{
Xin Yang and Periklis Petropoulos \\ Optoelectronics Research Centre, University of Southampton, Highfield, Southampton, SO17 1 BJ \\ xy1v07@orc.soton.ac.uk
}

\begin{abstract}
We present the nonlinear generation of ultra-flat broadband spectra suited to high capacity communication signals. A spectrum covering the full C-band (3-dB bandwidth of $35 \mathrm{~nm}$ ) is generated in an adaptable and power-efficient fashion.

OCIS codes: (060.4370) Nonlinear Optics, Fibres; (060.5530) Pulse Propagation and Temporal Solitons
\end{abstract}

\section{Introduction}

The emergence of new techniques for encoding and transmitting information in a spectrally efficient manner over optical fibers calls for the development of stable, broadband sources with accurately controllable characteristics. Of particular interest is the development of broadband frequency combs for use in applications, such as optical orthogonal frequency division multiplexing (OFDM) [1]. Such applications require the generation of discrete line spectra with as little power variations over their entire spectrum as possible. Kerr effects in optical fibers are frequently used to broaden the spectra of pulsed lasers, the tailoring of which relies on the use of optical fibers with specially designed dispersion properties. To this end, carefully engineered microstructured fibers offering suitable dispersion and nonlinear characteristics are extensively used for the generation of smooth broadband spectra [2].

Rather than relying on the special characteristics of highly nonlinear fibers (HNLFs), one can just shape the broadband spectrum after nonlinear propagation [3]. This technique can only remove portions of power through filtering a signal which has previously been amplified to stimulate sufficient nonlinearities in the HNLF. Meanwhile, the resulting bandwidth depends directly on the characteristics of the filter/shaper itself. We have recently proposed a more power-efficient approach in which the source spectrum is shaped prior to the nonlinear element (and amplifier), so that after nonlinear propagation a spectrum with desired characteristics is obtained. Potentially, this approach allows the manipulation of optical spectra with a spectral range that exceeds the operating bandwidth of the pulse shaper. A spectrum exhibiting a $3-\mathrm{dB}$ bandwidth of $13 \mathrm{~nm}$ with a maximum peak-to-trough ripple of $3 \mathrm{~dB}$ was achieved through this approach [4]. The demonstration was based on a pulse shaper based on liquid crystal on silicon (LCOS) technology [5], providing precise control over both the spectral amplitude and phase of the pulsed laser signal, as well as the capability to dynamically change the filtering characteristics. In order to further improve the flatness of the broadened spectrum, an adaptive pulse shaping system exploiting a simple evolutionary algorithm was subsequently included in the spectral synthesis technique. This improved the results significantly and a spectrum exhibiting a $3-\mathrm{dB}$ bandwidth of $11 \mathrm{~nm}$ with a $7-\mathrm{nm}$ central region where the power fluctuations were less than $0.5 \mathrm{~dB}$ was obtained [6].

In this work, we present two significant improvements to the previously reported results. We have targeted the generation of an ultra-flat frequency comb covering the full C-band, i.e. a spectral broadening of more than 10 times the bandwidth of the initial source spectrum. To achieve this, we developed a more sophisticated algorithm for the adaptive shaping process, based on the covariance matrix adaptation evolution strategy (CMA-ES) [7]. We report the generation of a spectrum with a 35-nm 3-dB bandwidth (and a 0.7-dB bandwidth of $26 \mathrm{~nm}$ ).

\section{Adaptive Pulse Shaping System Based on CMA-ES}

In our previous work reported in Ref. [6], a two-membered evolution strategy (ES) algorithm was used in the adaptive pulse shaping system, which is the simplest and most efficient algorithm in the ES family. A drawback of this algorithm is that the optimization process is likely to be trapped at a local minimum. In contrast, CMA-ES, the application of which we consider in this work, allows the optimization over a broader search space, thus making it more robust against local minima. In this section, the general principle of the pulse shaping system based on CMAES is described. A detailed description of CMA-ES can be found in Ref. [7].

Our adaptive pulse shaping system comprises a programmable phase and amplitude pulse shaper, followed by the nonlinear broadening stage (typically an amplifier and a HNLF). The CMA-ES algorithm monitors the spectrum $S_{\text {meas }}$ at the output of the system and adjusts the transfer function (both amplitude and phase) of the pulse shaper with the aim of minimizing the misfit function $M F$ between $S_{\text {meas }}$ and the target spectrum $S_{\text {target }}$ :

$$
M F^{2}=\int\left(S_{\text {meas }}-S_{\text {target }}\right)^{2} d \omega / \int S_{\text {meas }}^{2} d \omega .
$$


In the optimization process of CMA-ES, the shaping functions of both amplitude and phase are represented by a weighted summation of first kind Chebyshev polynomials of $10^{\text {th }}$ order. CMA-ES is optimizing the 11 weights of the polynomials. The starting vector of weights for the first generation of CMA-ES is a vector of all zeros. Thus, the pulse shaper is initially programmed to be a dispersionless bandpass filter of $20 \mathrm{~nm}$ bandwidth. In the mutation process, $\lambda=13$ vectors of weights are generated by adding a random (Gaussian distributed) vector to the starting vector of weights. The variance of the Gaussian distributed vector is determined by the step size of CMA-ES, which is self-adapted. Subsequently, each of the corresponding 13 transfer functions is fed into the pulse shaper and their $M F$ values are calculated. The vectors of weights are ranked according to their $M F$ values. Then, $\mu=6$ best out of the 13 vectors of weights are selected into the weighted recombination process, in which larger weights are assigned to better vectors of weights [7]. The resulting vector of weights becomes the starting vector of weights for the next generation. The algorithm is terminated when the value of $M F$ has converged.

By testing a large number of transfer functions $(\lambda)$ in each generation a broader search space can be explored during the optimization process. Meanwhile, the weighted recombination, in which the best transfer function is rewarded with the largest weight, keeps the transfer function evolving towards the optimum direction. When the process is trapped at a local minimum, the self-adaptation transition of the step size can rescue the optimization process. With all these advantages, the CMA-ES can approach the global optimum transfer function gradually.
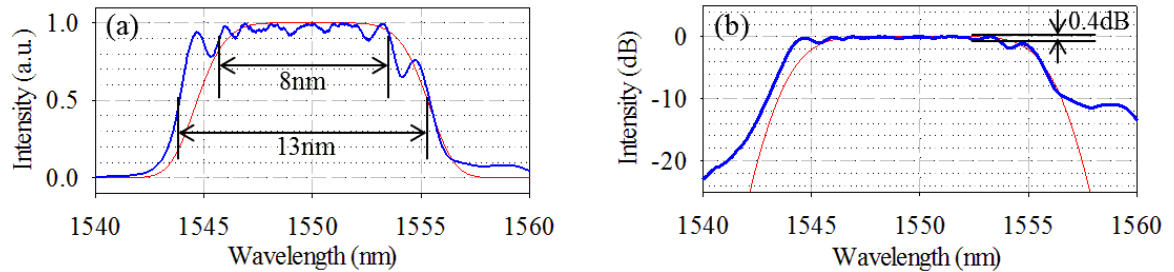

Fig. 1 Generated 13-nm SPM spectra with the application of the adaptive system using CMA-ES. (a) Linear Scale, (b) Logarithmic Scale. Red traces: target spectra, blue traces: measured spectra.

In order to test the performance of CMA-ES, we first substituted the two-membered ES with the CMA-ES in the adaptive pulse shaping system used in Ref. [6]. We confirmed that the quality of the generated spectrum was further improved, now exhibiting a $3-\mathrm{dB}$ bandwidth of $13 \mathrm{~nm}$ with a central region of $8 \mathrm{~nm}$ where the fluctuation is less than $0.4 \mathrm{~dB}$ (Fig. 1). We therefore used the adaptive pulse shaping system based on CMA-ES in a second experiment aiming at a significant scaling of the generated spectral bandwidth.

\section{Generation of Ultra-flat Broadband Frequency Comb}

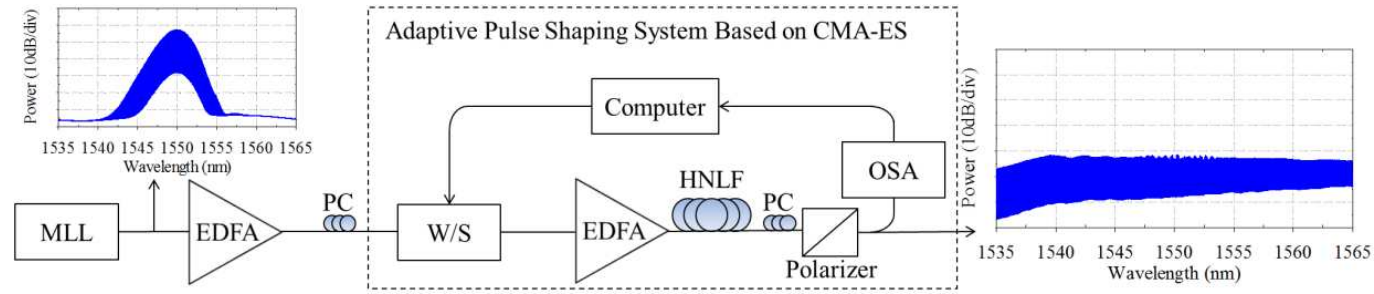

Fig. 2 Experimental Setup for the nonlinear generation of the ultra-flat broadband spectrum. PC: Polarization controller; EDFA: Erbium Doped Fiber amplifier; OSA: Optical Spectrum Analyzer. Insets show the high resolution $(0.01 \mathrm{~nm})$ measurements of the initial MLL pulses and the generated frequency comb.

Fig. 2 shows the experimental setup for the nonlinear generation of the ultra-flat broadband frequency comb. The output of the mode-locked laser (MLL) is 2 ps Gaussian pulses at $1550 \mathrm{~nm}$ with a repetition rate of $10 \mathrm{GHz}$. After a pre-amplification stage, the pulses were fed into the programmable phase and amplitude filter (Finisar Waveshaper $\mathrm{W} / \mathrm{S}$ ). The dynamic range of the $\mathrm{W} / \mathrm{S}$ is $35 \mathrm{~dB}$ and $2 \pi$ for the amplitude and phase shaping, respectively. The insertion loss is $5 \mathrm{~dB}$ and its individual spectral features can be programmed at $1 \mathrm{GHz}$ steps, much finer than the spectral line spacing of the $10 \mathrm{GHz}$ source itself. The shaped pulses were amplified to $32 \mathrm{dBm}$ and launched into a $500 \mathrm{~m}$ long HNLF with a nonlinear coefficient of $19 / \mathrm{W} / \mathrm{km}$, loss of $2 \mathrm{~dB} / \mathrm{km}$ and a dispersion of $-0.87 \mathrm{ps} / \mathrm{nm} / \mathrm{km}$ at $1550 \mathrm{~nm}$. The output of the HNLF was monitored by an OSA, placed after a polarizer, which was included to ensure that the generated frequency comb had a single polarization, as required in most applications. This measurement was also used as the feedback signal for the adaptive pulse shaping system implemented at the computer.

The target of our experiment was to generate an ultra-flat spectrum with a $35 \mathrm{~nm} 3$-dB bandwidth. Since the parameter we were mainly interested in was the flatness at the top of the generated spectrum $S_{\text {meas }}$, the target spectrum $S_{\text {target }}$ was simply a line of uniform spectral density across a $30 \mathrm{~nm}$ bandwidth centered at $1550 \mathrm{~nm}$. 

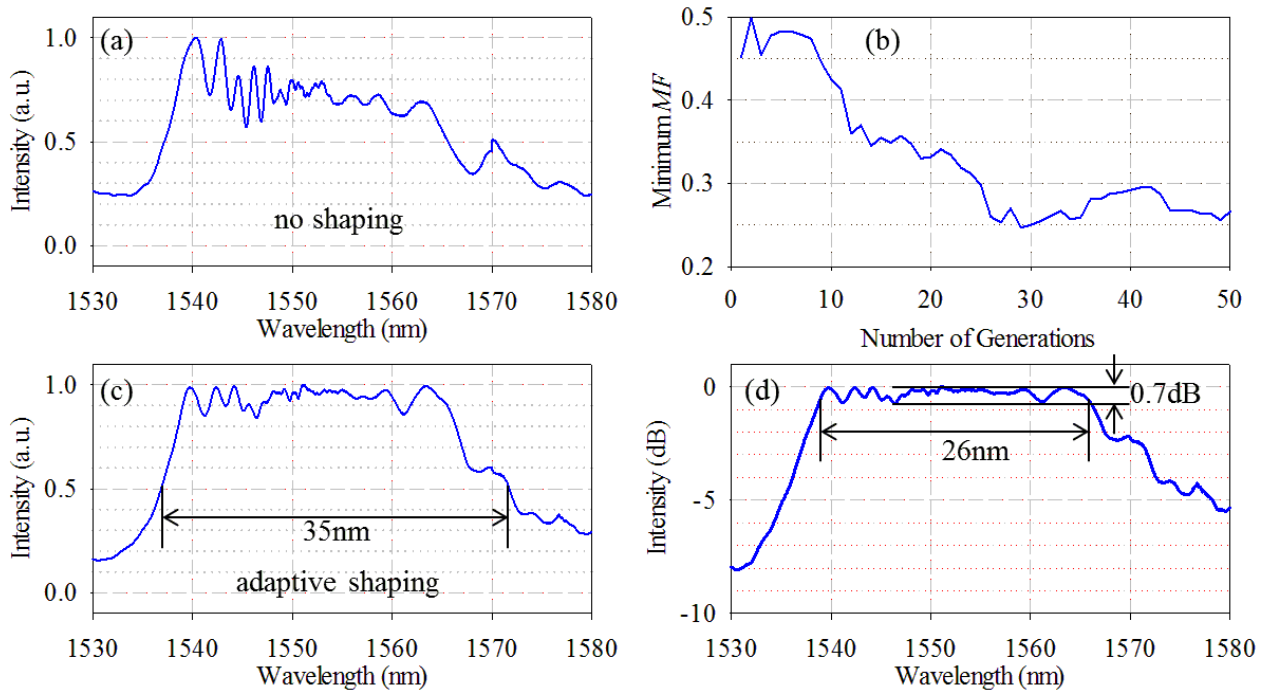

Fig. 3 SPM spectra generated from (a) 2ps Gaussian pulses, and (c and d) after the application of the adaptive system using the CMA-ES in linear and logarithmic scale respectively (resolution $\mathrm{b} / \mathrm{w}=0.2 \mathrm{~nm}$ ). (b) Evolution of the minimum $M F$ value after each generation in the CMA-ES algorithm.

In order to demonstrate the effectiveness of our technique, we first present in Fig. 3(a) the experimentally generated spectrum at the output of the HNLF when no shaping had been applied. The spectrum exhibits a multitude of spectral peaks with large fluctuations. We next consider the system when CMA-ES-based adaptive shaping was applied. Fig. 3(b) plots the minimum $M F$ value versus the number of generations - the minimum $M F$ value was reduced by half within 30 generations (390 iterations). The final spectra we obtained are presented in Fig. 3(c) (on a linear scale) and Fig. 3(d) (on a logarithmic scale); the traces show significant improvement in the spectral flatness. A 3-dB bandwidth of $35 \mathrm{~nm}$ was achieved, and the power variations in a central region of $26 \mathrm{~nm}$ were less than $0.7 \mathrm{~dB}$. High resolution measurement results of both generated ultra-flat frequency comb and the spectrum of the initial MLL pulses are presented in the insets of Fig. 2. The generated ultra-flat frequency comb has the same line spacing as the source pulses. Thus, in the $26 \mathrm{~nm}$ flat central region, 325 spectral lines were accommodated. As the HNLF used in the experiment was not polarization maintaining (PM), the polarized frequency comb at the output could be prone to random changes of birefringence. To assess the stability of the system, we performed spectral measurements over a period of 90 minutes, and found the power variation of any of the frequency lines across the entire comb to be less than $1 \mathrm{~dB}$ during this time. We anticipate better overall stability to be achieved through the use of a fully PM system.

\section{Conclusions}

We have presented the nonlinear generation of an ultra-flat broadband spectrum based on adaptive pulse pre-shaping using CMA-ES in a HNLF. We have applied this technique to obtain a stable frequency comb covering the full Cband with a $0.7-\mathrm{dB}$ bandwidth of $26 \mathrm{~nm}$. The CMA-ES played a vital role in the generation process. We believe this technique can be used for the generation of densely spaced high signal-to-noise ratio OFDM carriers in high capacity communication systems.

\section{References}

[1] Hillerkuss, D., et al., 26 Tbit s(-1) line-rate super-channel transmission utilizing all-optical fast Fourier transform processing. Nature Photonics, 2011. 5(6): p. 364-371.

[2] Hooper, L.E., et al., All-Normal Dispersion Photonic Crystal Fiber for Coherent Supercontinuum Generation. 2010 Conference on Lasers and Electro-Optics (CLEO), 2010: p. CTuX4.

[3] Clarke, A.M., et al., Reconfigurable Optical Pulse Generator Employing a Fourier-Domain Programmable Optical Processor. Journal of Lightwave Technology, 2010. 28(1): p. 97-103.

[4] Yang, X., D.J. Richardson, and P. Petropoulos, Pulse Shaping-Assitsed Nonlinear Spectral Broadening, in The European Conference on Lasers and Electro-Optics and the XIIth European Quantum Electronics Conference (CLEO®/Europe-EQEC)2011: Munich, Germany. p. CI2.1 TUE.

[5] Baxter, G., et al., Highly programmable Wavelength Selective Switch based on Liquid Crystal on Silicon switching elements. 2006 Optical Fiber Communication Conference, 2006: p. OTuF2.

[6] Yang, X., D.J. Richardson, and P. Petropoulos, Nonlinear Generation of Ultra-flat broadened Spectrum Based on Adaptive Pulse Shaping, in The European Conference on Optical Communication(ECOC)2011: Geneva, Switzerland. p. We.7.A.2.

[7] Hansen, N. and A. Ostermeier, Completely derandomized self-adaptation in evolution strategies. Evolutionary Computation, 2001. 9(2): p. $159-195$. 